\title{
Amplitude of Probability Density Function (APDF) of vibration response as a robust tool for gearbox diagnosis
}

\author{
P.J. Rzeszucinski ${ }^{1}$, J. K. Sinha ${ }^{1, *}$, R. Edwards ${ }^{1}$, A. $\operatorname{Starr}^{2}$, B. Allen ${ }^{3}$ \\ ${ }^{1}$ School of MACE, The University of Manchester, Manchester, M13 9PL, UK \\ e-mail: pawel.rzeszucinski@postgrad.manchester.ac.uk, jyoti.sinha@manchester.ac.uk, \\ rodger.edwards@manchester.ac.uk \\ ${ }^{2}$ Cranfield University, Cranfield, Bedfordshire, MK43 0AL, UK \\ e-mail: a.starr@ cranfield.ac.uk \\ ${ }^{3}$ QinetiQ Ltd., Farnborough, Hampshire, GU14 0LX, UK \\ e-mail: $\underline{\text { ballen2@qinetiq.com }}$ \\ *Corresponding author
}

\begin{abstract}
A 'Go' or 'No Go' assessment is a safety requirement for quick and robust estimation of the condition of gearboxes used in helicopters and other critical machines. A range of vibrationbased Condition Indicators (CIs) has been developed to meet this requirement. CIs are compared automatically with pre-set threshold values representing a healthy system, so that the health of the gearbox can be assessed and diagnosis made where necessary. The use of kurtosis of the residual signal of the measured vibration data, computed as part of the "FM4" method, is widespread, because it is accepted as a good and reliable indicator. However it has been observed in some cases that FM4 may not show a continually increasing trend with the propagation of a fault. This behaviour may lead to improper assessment of the severity of the fault. Hence a new CI, based on the deviation in the normal Probability Density Function (PDF) of the measured vibration data, is suggested which demonstrates an increasing trend which is more robustly and monotonically correlated with the fault propagation.
\end{abstract}

Key words: Condition Monitoring, Vibration analysis, Gearbox diagnosis, Probability Density Function, Condition Indicator 


\subsection{Introduction}

Helicopter drive trains are extremely compact, which makes the task of performing proper inflight condition monitoring very difficult. Complex geometry, complicated stress states, crack growth patterns which are difficult to predict, and a very complex load history add to the extreme difficulty in assessing the condition of helicopter components [1]. In a comprehensive analysis of the cause of helicopter accidents, NASA observed that $38 \%$ of all accidents for single piston-engined helicopters were related to the drive trains to the main and tail rotors [2]. $27 \%$ of those accidents were due to main or tail rotor gearbox failure, where the direct source of failure was dominated by gear failures. Those types of malfunctions have often led to some very serious consequences including fatalities, for example the Eurocopter Super Puma accident (G-REDL) that took place in March 2009, where the post-crash preliminary investigation revealed that the accident occurred following a catastrophic failure of the main rotor gearbox which resulted in detachment of the main rotor head from the helicopter [3].

Although the science of vibration analysis forms the basis of a number of techniques capable of properly indicating a gear fault, most of them require specialist knowledge, offline analysis of data, advanced signal processing techniques and much user experience of data interpretation in order to determine if a gear fault has developed [4-19]. Due to the demanding requirements, these approaches may be considered inappropriate in a situation when a 'Go' or 'No Go' indication of gear health is required, when it must be quick, automatic, and easily interpreted, perhaps in-flight, by a non-specialist like a helicopter pilot. For this reason, one of the most widely used helicopter on-board mounted condition monitoring systems these days is the Health and Usage Monitoring System (HUMS) [20]. It has been specifically designed to monitor the condition of all safety-critical components operating in the helicopter. Where faults are detected by vibration, condition indicators (CIs) are calculated based on signal processing routines of measured vibration responses designed to output a single number for each CI, representing the condition of given monitored system. CIs are designed to react to various drive-train component faults, for example gear tooth cracking or bearing defects. CIs are compared with pre-set threshold values, which are established based on historical data and the values generated by the CI for the healthy condition. The crossing of the threshold level gives a clear and quick indication of fault progression within the system. 
There are many gearbox related CIs suggested in the literature. Rzeszucinski [21] gave a review of a range of CIs and compared their sensitivity and the performance on simulated and experimental datasets. An important trend in vibration-based condition monitoring of gears is to use the non-dimensional parameter "kurtosis" of the residual signal of the measured vibration data for health assessment, as part of a computed method CI called "FM4" [4-10], because it is observed to be a good and reliable indicator. The concept of the residual signal derived from the measured vibration signal is briefly discussed for better understanding. Considering a gearbox in a healthy condition, it is generally observed that there is some amount of unavoidable manufacturing and assembly imperfections, for example residual rotor unbalance, small misalignments in the shafts, and transmission error. Hence the vibration of the healthy gearbox often contains peaks related to the shaft rotational frequency (1x RPM) and the gear mesh frequency (GMF) and their harmonics as well. The GMF is defined as the product of the shaft RPM (1x) and the number of teeth on the gear mounted on the shaft [11]. As the gear tooth fault develops, short duration impacts modulate the vibration signal and generate shaft speed sidebands that appear around the GMF and its harmonics, hence changing the vibration responses and the signal's distribution [12-13]. The spacing of the sidebands is equal to the relevant shaft speed - drive or driven - depending on which shaft the faulty gear is mounted. Despite this phenomenon being a clear indicator of a gear fault, kurtosis may not respond to it immediately, due to the much stronger GMF and its harmonics masking the impulsive content of the signal. The development of FM4 was based upon this observation. Stewart [7] suggested removing the known frequency peaks related to the shaft RPM $(1 \mathrm{x}, 2 \mathrm{x}, \ldots)$ and the GMF and its harmonics $(1 \mathrm{xGMF}, 2 \mathrm{xGMF}, \ldots .$.$) from the vibration$ spectrum of original measured vibration signal. The remaining signal is called the "residual signal".

The advantage of such a residual signal is that, in the case of gear tooth failure, it only contains information generated by the impulsive nature of contact during the meshing action between two gears [14]. FM4 is generally capable of properly detecting a fault in its early stages, and its value continues to rise as long as the fault remains localized. It has been observed, however, that as the fault starts to broaden, interacting with the adjacent teeth, the value of kurtosis, and therefore FM4, start to drop [8, 15-18]. Such behaviour has the potential to influence heavily the robustness of automated condition monitoring systems, and to lead to some improper assessments being made as to the severity of the fault. Therefore, 
this behaviour may not be acceptable in many critical field applications since it could introduce some level of uncertainty about the actual condition of the monitored component. Hence a more robust method, showing a continually increasing trend with the fault propagation in the gearbox, is needed in addition to the existing CIs including the FM4 indicator, for reliable and accurate prediction of the fault presence and the stage of the fault severity.

In this paper a new CI has been proposed based on the changes in amplitude of the Probability Density Function (PDF) curve of a vibration acceleration response and applied on a set of experimental vibration data. The data used contains a full history of progressively deteriorating gear condition - from healthy condition until catastrophic failure. The paper presents the concept of the proposed new CI and its results, which have been compared with the FM4 indicator.

\subsection{Comparison of Vibration Signals for Healthy and Faulty Cases}

The residual signal from a healthy gear system, without the shaft related frequencies and the GMF and its harmonics, may be similar to a random signal. Hence a simulated normally distributed signal shown in Figure 1(a) has been considered as the residual vibration signal for the healthy gear system. The residual vibration signal is expected to contain the high energy impulsive responses in addition to the signal in Figure 1(a) due to the regular impact caused by faulty tooth/teeth mesh during rotation, for a faulty gear system. Figure 1(b) represents a typical simulated residual vibration signal for the faulty gear system. Figure 2 shows the histograms of both signals, which indicate significant similarity between the two signals at low amplitudes, but longer tails for the faulty system due to the high energy peaks in the signal. 

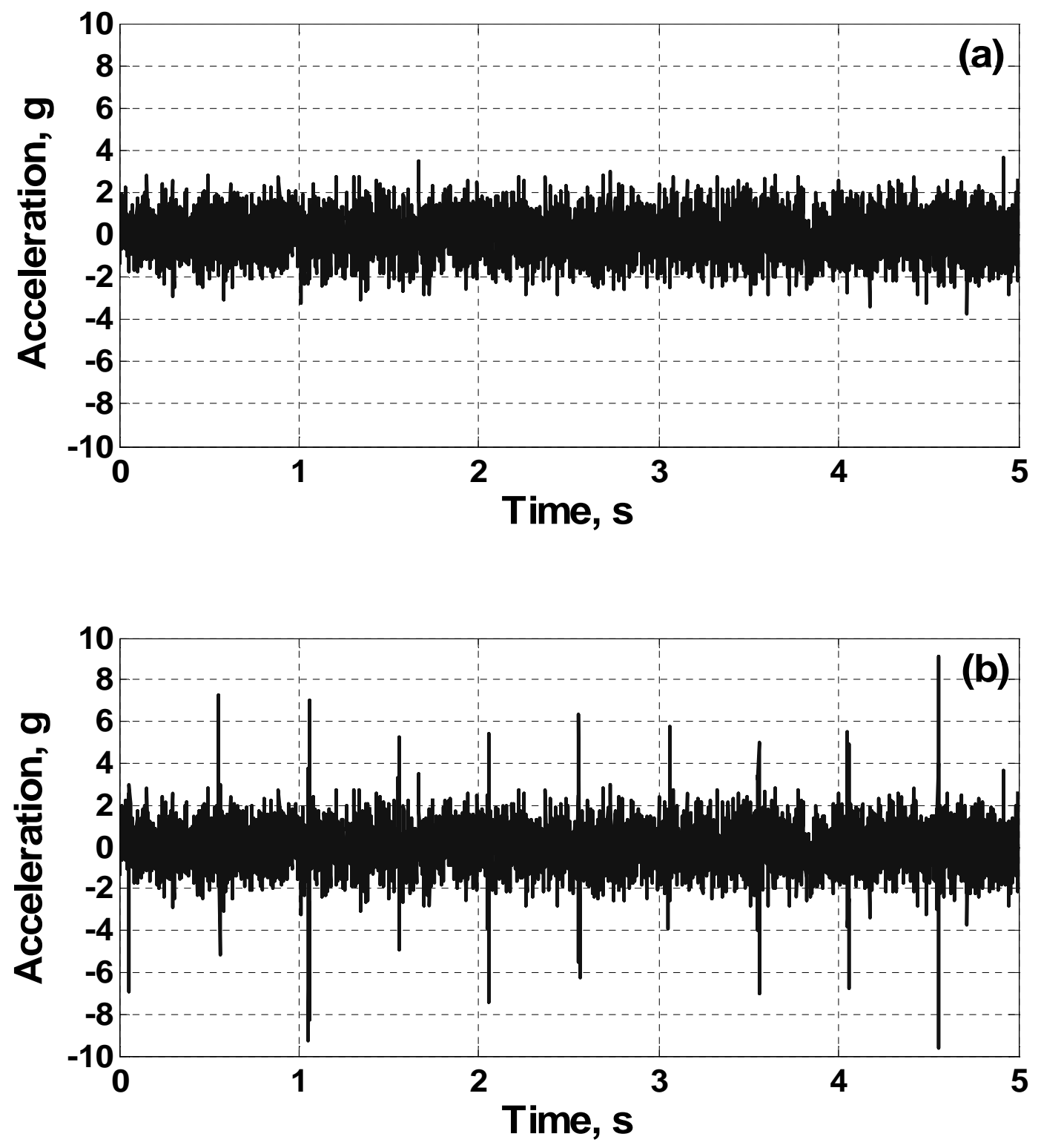

Figure 1 Typical simulated residual vibration acceleration response for a gearbox, (a) healthy condition, (b) faulty condition 

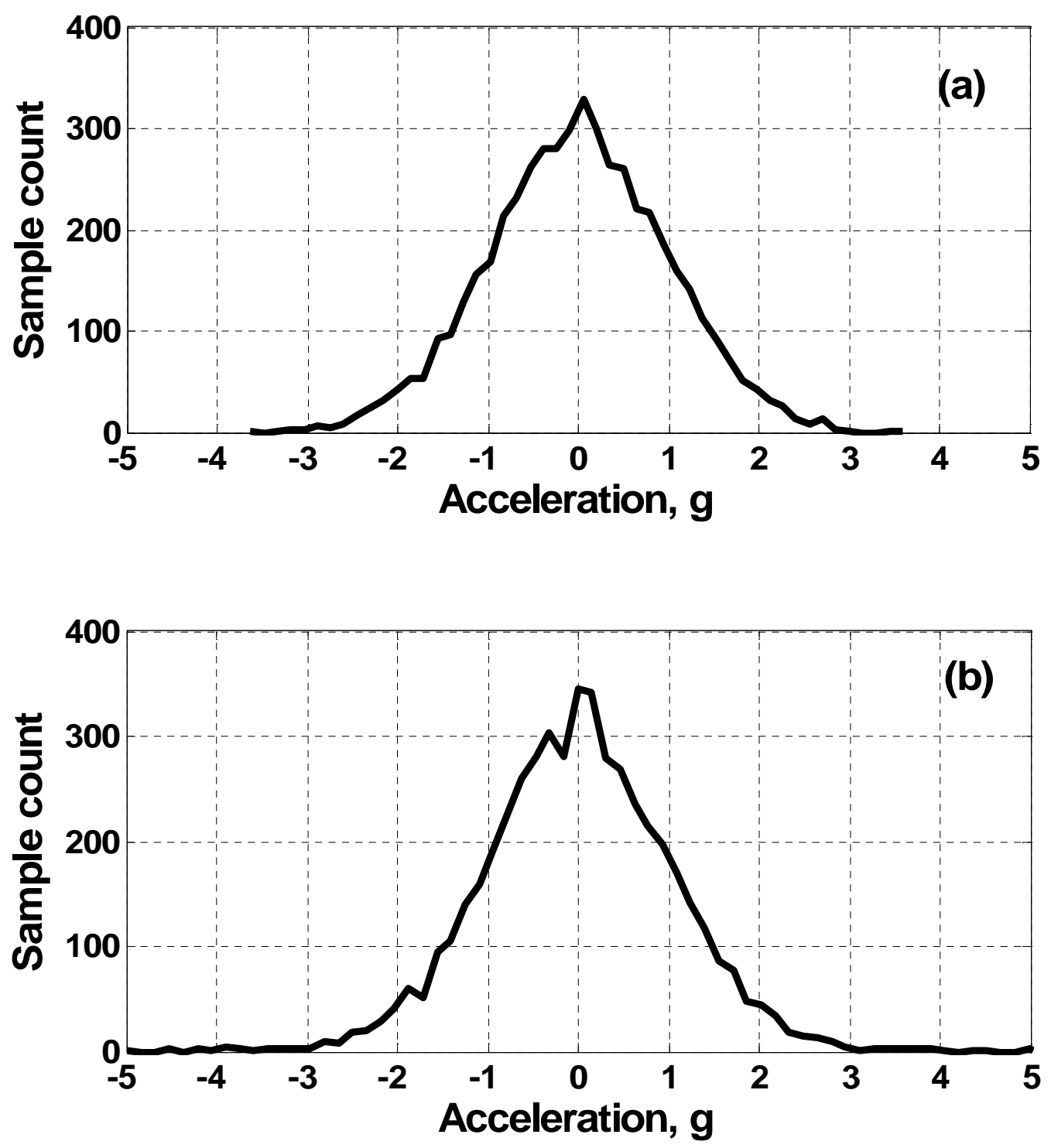

Figure 2 Typical histograms of the residual signals for a gearbox in Figure 1, (a) healthy condition, (b) faulty condition

These histograms can be presented on a normalised scale in the form of normal Probability Density Function (PDF) curves. The normal PDF for a signal $x(t)$ can be mathematically described as [22]:

$$
f(x)=\frac{1}{\sqrt{2 \pi} \sigma} \cdot e^{-\frac{(x-\mu)^{2}}{2 \sigma^{2}}}
$$


where $x(t)$ is a time domain signal, $\sigma$ is the standard deviation of $x(t)$ and $\mu$ is the mean value of $x(t)$. Figure 3 shows the normal PDF curves calculated from Equation (1) for the signals shown in Figure 1. A clear difference between the two simulated residual signals for the healthy and faulty gear system is observed through the PDF curves in Figure 2, i.e. a broader PDF curve with lower peak amplitude for the faulty system compared to the healthy system. The advantage of using the normal PDF for the data analysis is that it is normalised such that the area under the curve is always unity, and hence the actual vibration amplitude is not important. This means that the PDF curve for a healthy or a particular fault severity is expected to remain unchanged for different load conditions. This is because the gearbox system is generally assumed to be a mechanical system with a linear dynamic behaviour and hence the change in the load is expected to linearly affect the amplitude of the vibration response of the gear system. Hence this concept has been used for diagnosis of gears.

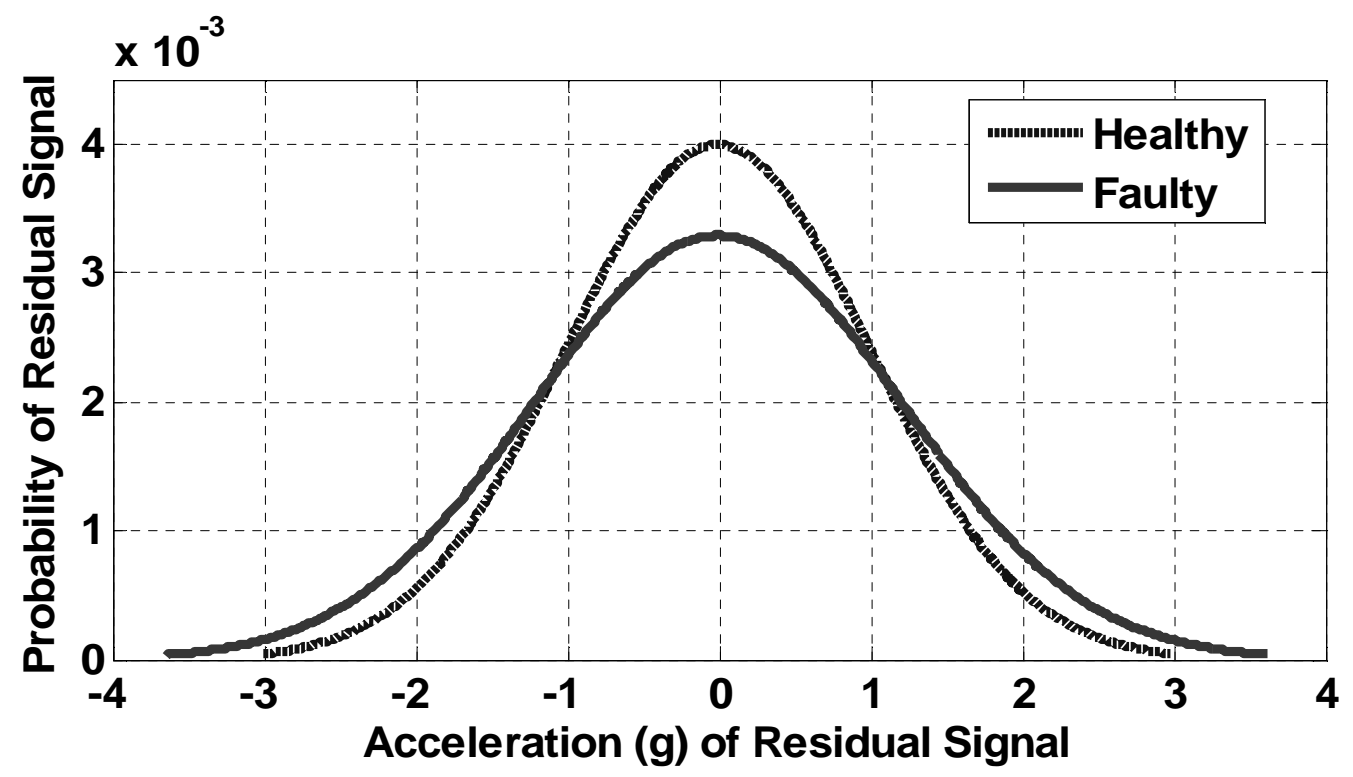

Figure 3 Typical normal PDF curves of the residual signals from Figure 1

\subsection{The Proposed CI and the Computational Approach}

The proposed CI is based on changes in the Amplitude of normal Probability Density Function (APDF) of the residual vibration signal. It is a non-dimensional parameter like FM4 and should be independent of load. The proposed CI can be calculated in two different forms, namely "reference based" and "reference free". 


\subsection{Reference free form}

Initially a reference free CI based on the APDF value, denoted "APDF $F_{R F}$ ", was proposed because it does not need any reference data from the healthy condition. $\mathrm{APDF}_{\mathrm{RF}}$ derived for the residual of the measured vibration acceleration signal $x(t)$ is mathematically described as:

$$
A P D F_{R F}=1-\max [f(x)]
$$

where $f(x)$ is the Normal Probability Density Function as per Equation (1). The proposed CI in Equation (2) is based on the assumption that the maximum amplitude of the PDF curve is expected to show a decreasing trend with the fault propagation. Since the area under the curve is always unity for all signals, the maximum value will always be less than 1 . Hence $\mathrm{APDF}_{\mathrm{RF}}$ is expected to show an increasing trend from the healthy condition to the propagating fault, and the value will not be more than 1 .

\subsection{Reference based form}

Another form of the CI is also proposed, which is a reference based CI denoted "APDF $\mathrm{RB}$ ". It uses the information contained in a vibration acceleration response of the healthy gear system as a reference value. All measurements are then compared against the reference value in order to determine if any potential changes appear in the signal. $\mathrm{APDF}_{\mathrm{RB}}$ is mathematically described as:

$$
A P D F_{R B}=\frac{\max \left[f(x)_{\text {HEALTHY }}\right]}{\max \left[f(x)_{\text {ACTUAL }}\right]}
$$

where $f(x)_{\text {HEALTHY }}$ is the normal PDF function calculated for a reference vibration signal generated by healthy pair of gears, $f(x)_{A C T U A L}$ is the normal PDF function calculated for the current measurements.

\subsection{Computational approach}

As explained in Section 1.0, the vibration spectrum of the healthy gearbox may contain the frequency peaks related to the shaft RPM $(1 \mathrm{x}, 2 \mathrm{x}, \ldots)$ and the GMF $(1 \mathrm{xGMF}, 2 \mathrm{xGMF}, \ldots)$. As the fault develops in the gearbox, additional peaks start appearing in the vibration spectrum as sidebands to the GMF and its harmonics, as shown in Figure 4. Figure 4 is an 
idealised simulated vibration spectrum of a faulty gearbox, and for the clear understanding of the features, the unit and amplitude scale is not shown in the figure. The presence of sideband peaks around the GMF and its harmonics in Figure 4 simply indicates that the short duration impacts due to the faulty tooth/teeth in the gearbox modulate the vibration responses and generate shaft RPM related sidebands. Hence the frequency components related to the shaft RPM and the GMF and their higher harmonics are likely to appear in the spectra of the vibration signals generated by both healthy and faulty gearboxes. For this reason, the common information contained in them can be considered as redundant, and can be removed from the vibration signal. The remaining vibration signal, after removal of the shaft speed related frequencies and the GMF and its harmonics, is called the "residual signal". It is achieved by performing the following processes [23].

i. Time Synchronous Averaging (TSA) of measured data to remove noise and enhance signal quality.

ii. Fourier Transformation of the TSA signal. The spectrum of the TSA signal for a faulty gearbox may be similar to Figure 4, containing clear peaks at the shaft RPM (1X), the GMF and its harmonics (1xGMF, 2xGMF...) and sidebands around GMF and its harmonics.

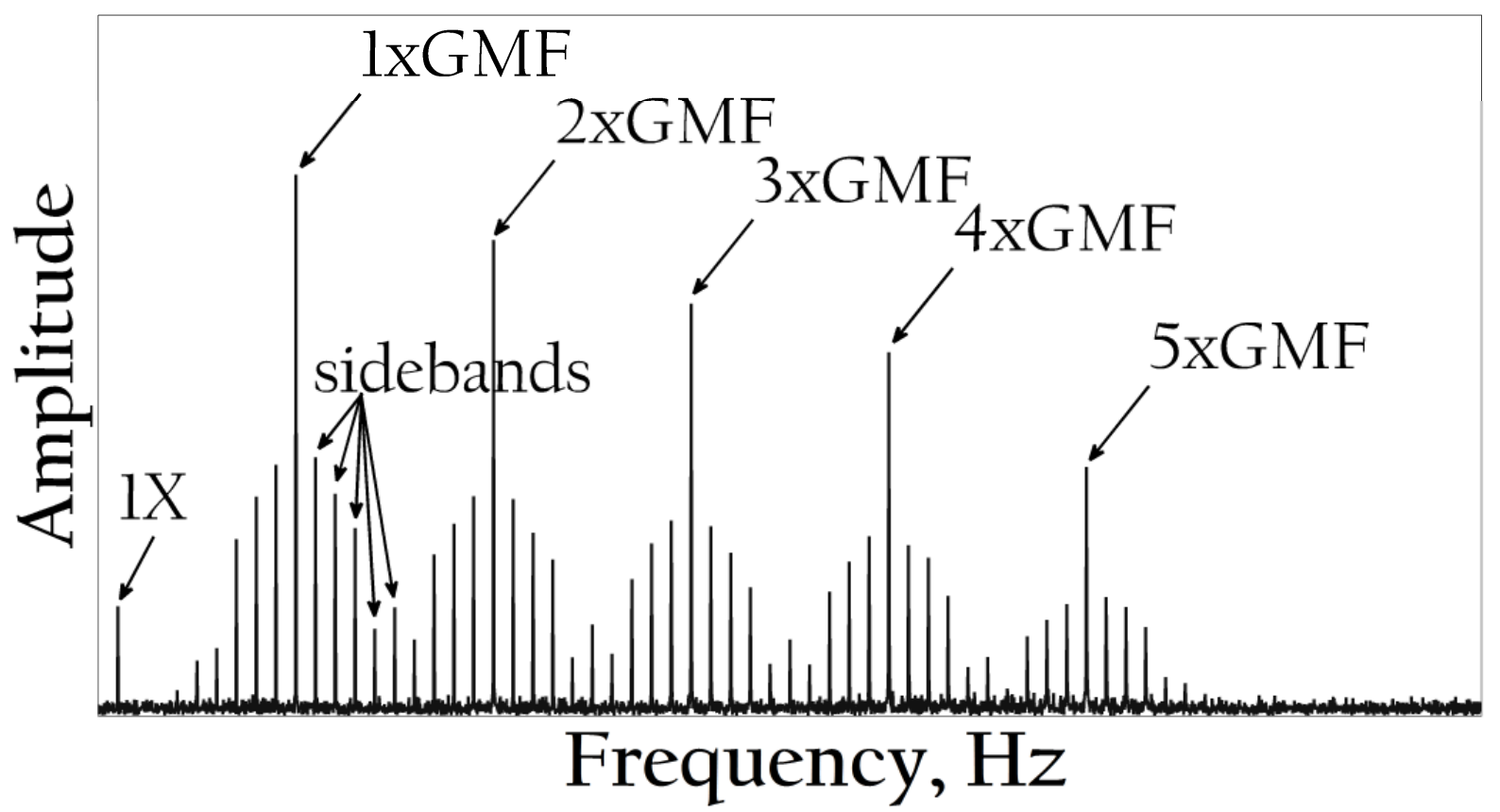

Figure 4 Idealised vibration spectrum of a faulty gearbox 
iii. Removal of components related to shaft speed, GMF and their harmonics from the spectrum. Those components are expected to appear in the vibration signals generated by healthy gearbox as well, therefore they have been removed to create the residual spectrum as shown in Figure 5.

iv. Inverse Fourier Transformation of the residual spectrum to generate the residual signal in the time domain;

Once the residual signal has been created, the PDF can be calculated according to Equation (1). This can then be followed by calculation of the proposed CIs in Equations (2) and (3).

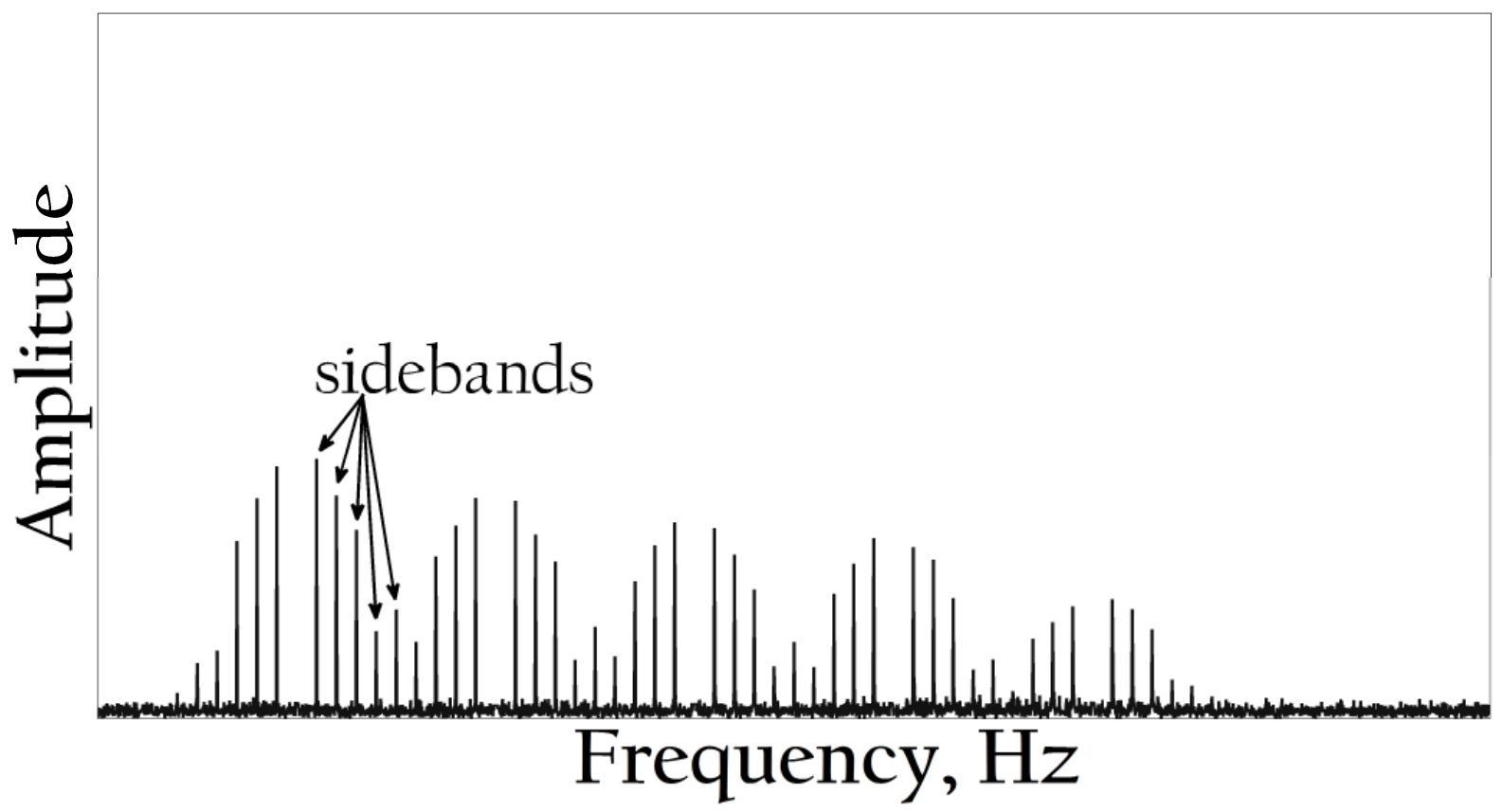

Figure 5 Idealised residual spectrum generated from Figure 4 for a faulty gearbox

\subsection{Experimental data}

The data were collected by the U.S. Navy's Helicopter Transmission Test Facility (HTTF), located at the Naval Air Warfare Center, Patuxent River, Maryland as part of the Helicopter Integrated Diagnostic System (HIDS) programme [24]. The test rig was a SH-60 Sea Hawk helicopter drive train. The objective of the test was to force gear fault propagation from a condition of good working order to a point of near catastrophic failure. The gear used in the test rig was a spiral bevel gear located in the Intermediate Gearbox (IGB). Figure 6 shows a simplified cross sectional view of the IGB with the accelerometer location from [24]. 
A crack was forced to propagate along a specific path along the length of the root and down into the web of the gear. In order to achieve this, two small notches were seeded on the gear prior to the test. One notch was located near the toe of the tooth, and the second near the centre, where the modelled load pattern indicated maximum load for a healthy gear. Both notches were located at the root of the gear tooth and both were parallel to it.

The test was conducted at a constant torque and constant speed, with manual inspections assessing the condition of the gear approximately every 2 hours during the entire test period. There were a total of 4 inspections carried out after Sample 25, 46, 57 and 74. Each inspection consisted of removing the gear from the test rig, removing the lubricant and performing a number of non-destructive tests in order to assess the condition of the gear.

The datasets comprise a total of 85 samples. The test was stopped after 85 samples due to a severe gear crack. Inspection revealed that the fault initiated from the tooth root, extended through the gear web and stopped at a bearing support diameter. A photograph of the gear taken after the test is shown in Figure 7.

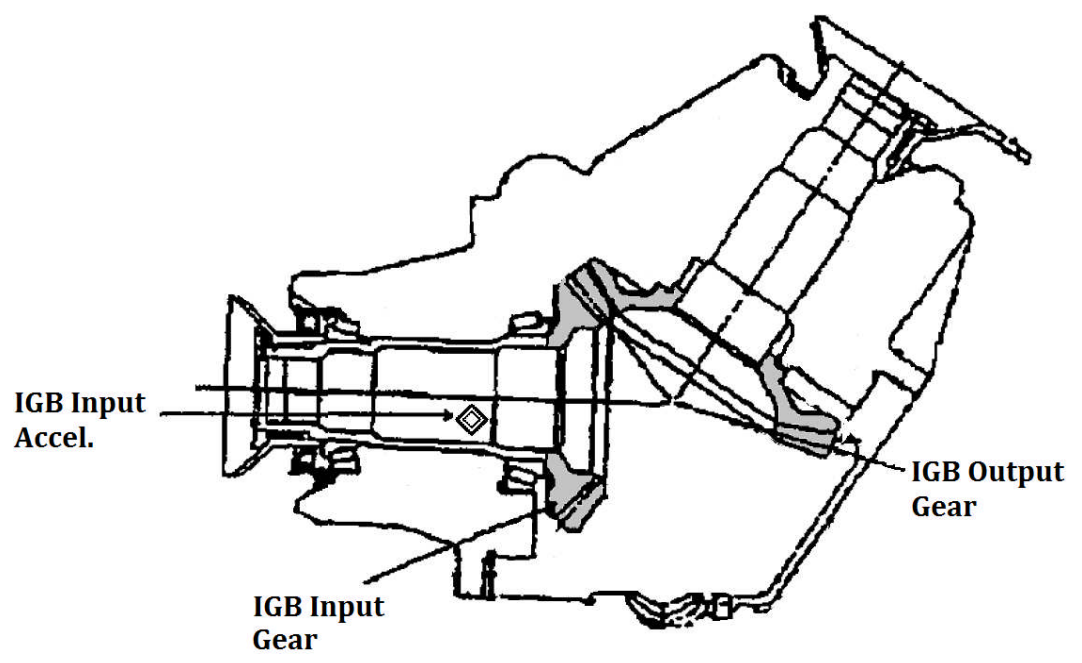

Figure 6 A simplified cross sectional view of the Intermediate Gearbox [24] 


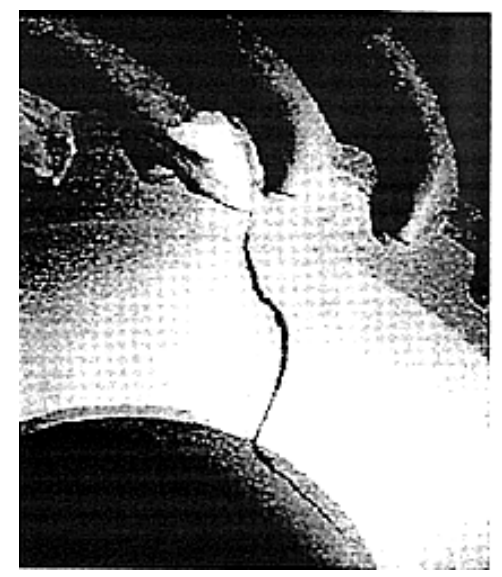

Figure 7 A severe gear crack that led to termination of the test [24]

Data files were recorded every 5 minutes and each sample acceleration response contains 30 seconds of raw vibration acceleration data. The sampling rate of the system was $100 \mathrm{kHz}$. For the present analysis, data were used from the accelerometer mounted on the IGB input bearing pedestal. It is also important to note that the data available for analysis were in the same scale for all tests, however the mechanical unit was not disclosed. Hence, the unit of the acceleration scale in all the figures related to this experimental dataset is not shown.

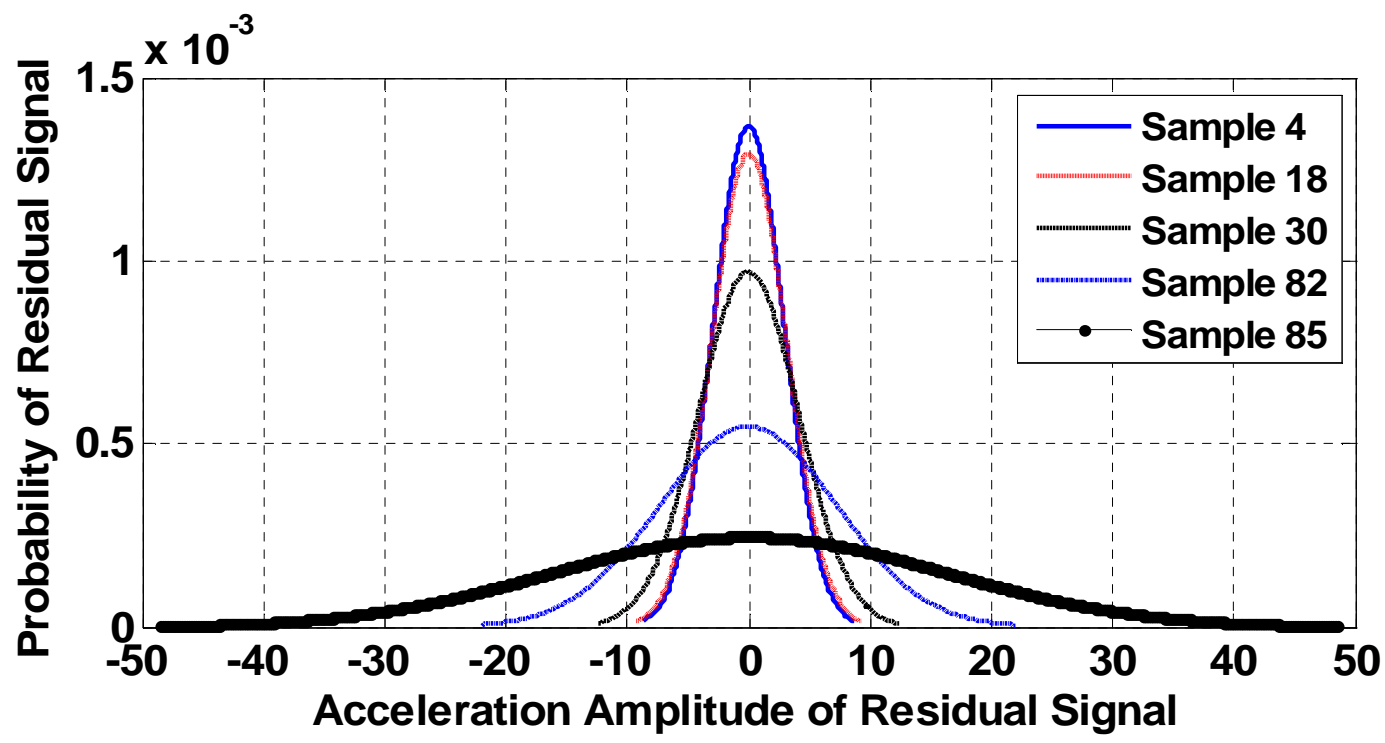

Figure 8 Some typical normal PDF curves of different samples of the dataset 


\subsection{Analysis and observations}

Initially the residual signals for the 85 samples in the datasets were extracted according to the steps discussed in Section 3.3, and the normal PDF functions were calculated using Equation (1) for the residual signals. Error! Reference source not found. presents a comparison of chronologically arranged normal PDF plots for a few typical samples from the dataset. It can be seen that the normal PDF curve for the healthy gear condition (Sample 4) has the highest maximal value. The gear fault started to develop at Sample 18 which is represented by a decrease in the maximal value of the normal PDF curve. As the gear fault progressed, the maximal value of the normal PDF curves decreased accordingly. It is clearly evident from Error! Reference source not found. that as the gear fault develops, the tails of the signal's amplitude distribution broaden, causing the value of the central point of the normal PDF plot to decrease. The explanation for this behaviour is that as the gear fault develops and propagates further, the dynamic behaviour changes progressively, thereby increasing the side band frequency components and their amplitudes. This in turn eventually leads to spread of the amplitude distribution tails and, at the same time, the decrease in the maximal value of the PDF curve because the area under PDF of normally distributed signal is always equal to 1 [22].

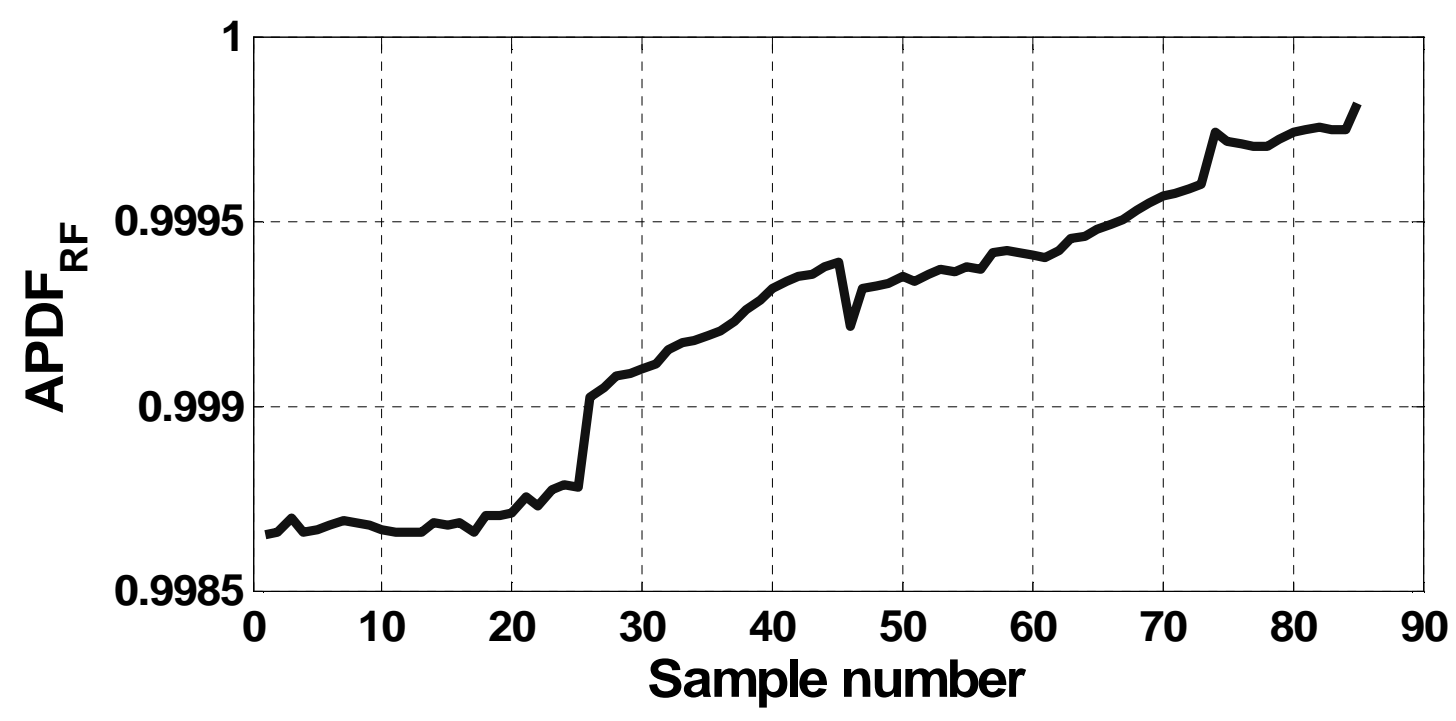

Figure 9 Variation of $\mathrm{APDF}_{\mathrm{RF}}$ with fault propagation 
The maximum values of the calculated PDF curves for all samples in the dataset were then used to calculate the proposed CI, $\mathrm{APDF}_{\mathrm{RF}}$, shown in Equation (2). The typical trend of $\mathrm{APDF}_{\mathrm{RF}}$ is shown in Figure 9. As observed from Figure 9, the value of $\mathrm{APDF}_{\mathrm{RF}}$ remains nearly constant up to Sample 17 and then slightly increases at Sample 18, confirming the initiation of the fault, and then shows almost continuous increasing trend with the fault propagation. Hence the observations of $\mathrm{APDF}_{\mathrm{RF}}$ are definitely encouraging and show the potential in the proposed method. However, the separation between the healthy and the faulty condition is very small, and hence $\mathrm{APDF}_{\mathrm{RF}}$ may not be useful for field applications.

Another proposed CI, $\mathrm{APDF}_{\mathrm{RB}}$ as shown in Equation (3), was calculated for all samples in the dataset and its results are shown in Figure 10. Once again, the plot shows an almost continuously rising trend as a function of the gear fault deterioration process. Here again, the increase in the value of the $\mathrm{APDF}_{\mathrm{RB}}$ begins at Sample 18, confirming the initiation of the fault, but the increase is very small. Thereafter the $\mathrm{APDF}_{\mathrm{RB}}$ output continues to rise until the test was terminated. In addition to this observation, the separation between the values generated for healthy and faulty gear is now large enough to discriminate and diagnose the condition, which makes $\mathrm{APDF}_{\mathrm{RB}}$ useful for field applications. The only requirement is to know the reference data, but this is hardly a limitation for the proposed CI, considering the advantages shown. Besides, it is common for condition monitoring techniques to require reference data for a base line.

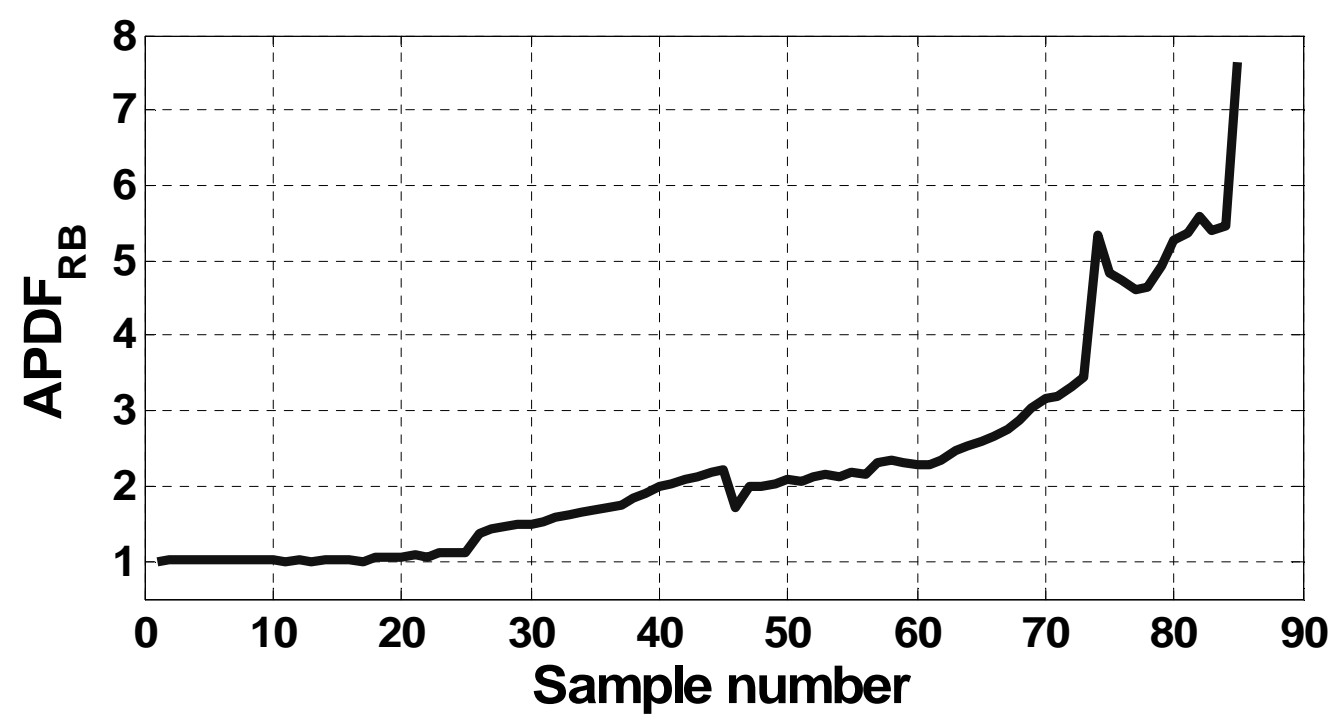

Figure 10 Variation of $\mathrm{APDF}_{\mathrm{RB}}$ with fault propagation 


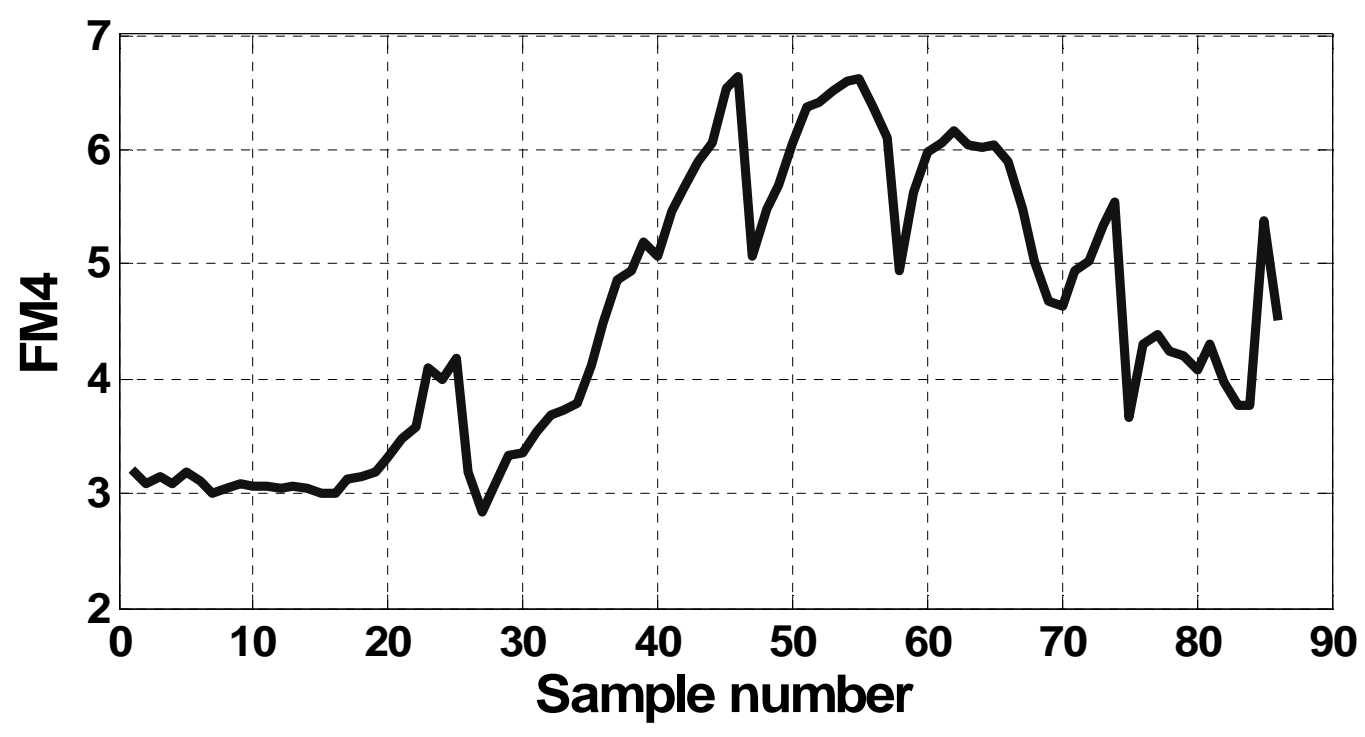

Figure 9 Variations of FM4 with fault propagation

\subsection{Performance comparison of $\mathrm{APDF}_{\mathrm{RB}}$ and the FM4 method}

For the purpose of comparison, the FM4 method has also been applied on the dataset provided by the HTTF and the result is shown on Figure 9. FM4 values remain stable up to Sample 18 when tooth damage has been initiated and, as a consequence, a sharp increasing trend is observed in the next few samples, which clearly indicates the potential of FM4 as a good indicator of the fault at a very early stage. However the value drops below the healthy level at Sample 25. Thereafter, FM4 shows an increasing trend until Sample 46, and then a decreasing trend until the end of the test, with some oscillation. This observation clearly indicates the FM4 may be good indicator at the early stage of the fault but it may not show a continuous and monotonically increasing trend with advancing fault propagation. This may produce misleading results in field observations.

It has been reported that the drop has been due to the damage spreading from a single tooth to several teeth [24]. Such behaviour of FM4 is not unique to the present dataset, and a number of studies reported that the values of FM4 drop as the fault transits from being localised, to a distributed fault affecting a higher number of teeth. As the gear fault develops from being localised, to more widely distributed, the generated vibration acceleration signal becomes less impulsive, and transforms into a more complex signal containing higher energy and more widely distributed frequency components. This in turn reduces the peaked nature of the 15 
signal's amplitude distribution which causes the value of the FM4 to drop. The study by Zakrajsek et al. [17] has also observed a similar phenomenon, which is shown in Figure 10. Here again, the FM4 indicator remains stable up to Sample 110, when tooth damage has been detected and, as a consequence, the values increase. However, shortly after that FM4 drops. Similarly to the previous case, it has been reported that the drop occurred due to the damage spreading from one, to a higher number of teeth.

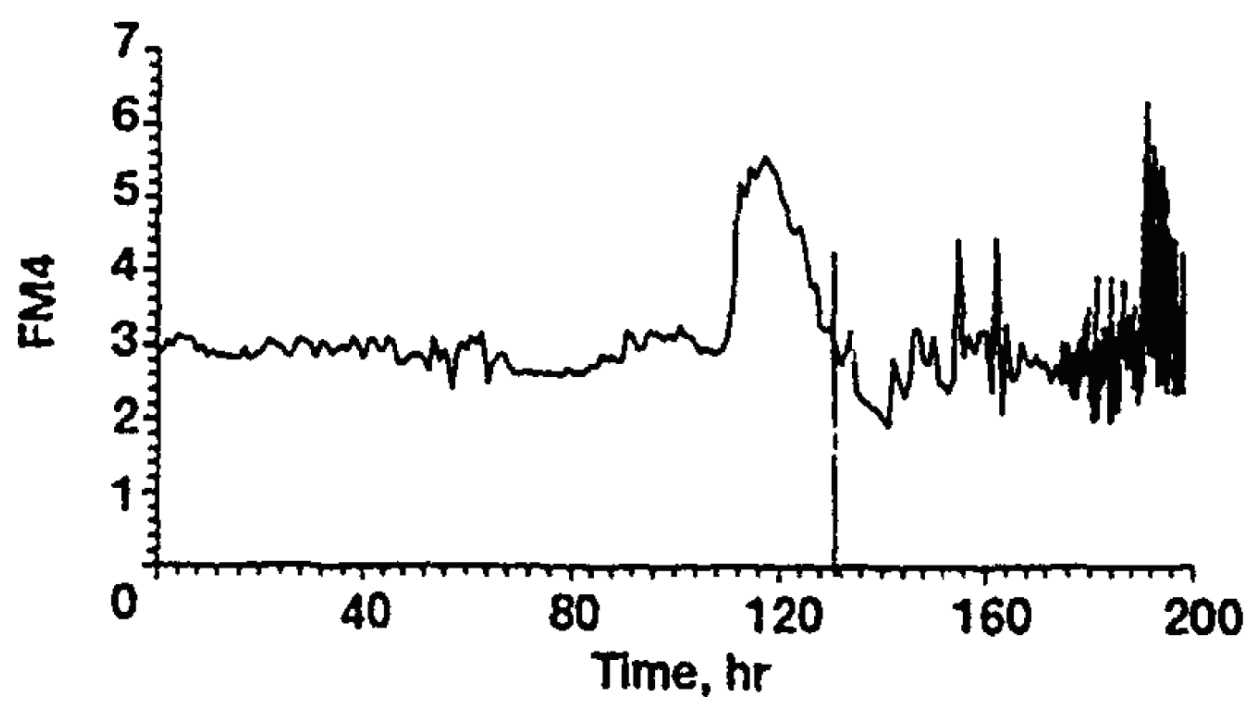

Figure 10 Variations of FM4 with fault progress [17]

\subsection{Concluding Remarks}

There is need for a "Go" or "No Go" type of indicator in some specific critical gear system applications so that it becomes useful tool for quick decision making. There is a number of such condition indicators (CIs) which are already in use and the FM4 indicator is observed to be the most reliable indicator so far for this purpose. However it has been reported a number of times that FM4 fails to show an increasing trend with propagation of gear fault, although it may be very sensitive at the early stage of the fault. Hence a new CI has been proposed, the APDF indicator, based on the amplitude of the normal Probability Density Function of the vibration acceleration signal. APDF has been proposed in two versions: reference free $\left(A P D F_{R F}\right)$ and reference based $\left(A P D F_{R B}\right)$. The results of both the reference free and reference based APDF CIs showed an increasing trend, not only with the initiation of the fault, but towards its further propagation, which is a distinctly positive feature for the diagnosis of fault 
propagation in a gear system. However, it is important to note that the separation between the healthy and the faulty condition is very small for the reference free $\mathrm{CI}, \mathrm{APDF}_{\mathrm{RF}}$, and hence it may not be useful for field applications. However, the separation between the values generated for healthy and faulty gears is large enough to discriminate and diagnose confidently for the reference based $\mathrm{CI}, \mathrm{APDF}_{\mathrm{RB}}$. Hence the $\mathrm{APDF}_{\mathrm{RB}}$ is useful for field applications. The only requirement is to know the reference data from the healthy condition, but this is hardly a limitation to the proposed CI, because most techniques need comparison to healthy data, and the CI has distinct advantage, particularly for safety critical applications. The results for $\mathrm{APDF}_{\mathrm{RB}}$ were more robustly and monotonically correlated to fault propagation than FM4 in the samples analysed.

Moreover, it is also important to note that the distinct level of the fault detection at the early stage is slightly delayed for $\mathrm{APDF}_{\mathrm{RB}}$ when compared with the FM4 results for the dataset in this study. This may be an advantage because of FM4's tendency to observe early faults, before subsiding. Hence $\mathrm{APDF}_{\mathrm{RB}}$ needs to be tested further on different experimental data to enhance the confidence level, and/or may be used alongside FM4 for more reliable and robust diagnosis.

\section{Acknowledgements}

The authors gratefully acknowledge the support of QinetiQ Ltd and the Engineering and Physical Science Research Council (EPSRC), and also the U.S. Navy's Helicopter Transmission Test Facility (HTTF), located at the Naval Air Warfare Center, Patuxent River, Maryland for providing the data for the analysis.

\section{References}

1. Symonds N, Pitt C. Military helicopters: Have the seeds of the future been already shown? Engineering Failure Analysis 2006; 13: 493 - 515.

2. Harris FD, Kasper EF, Iseler LE. U.S. Civil Rotorcraft Accidents, 1963 Through 1997. Ames Research Center 2000; NASA/TM-2000-209597.

3. Air Accidents Investigation Branch (AAIB) - Initial Report 2 2009; AAIB Ref: EW/C2009/04/01. 
4. Vecer P, Kreidl M, Smid R. Condition Indicators for Gearbox Condition Monitoring Systems. Acta Polytechnika 2005; 45(6): 35-43.

5. Samuel PD, Pines DJ. A review of vibration-based techniques for helicopter transmission diagnostics. Journal of Sound and Vibration 2002; 282: 475 - 508.

6. Stewart RM. Some Useful Data Analysis Techniques For Gearbox Diagnostics. Proceedings of Meeting on Applications of Time Series Analysis 1977; 18: 18.1-18.19.

7. Hochmann D, Bechhoefer E. Gear Tooth Crack Signals and their Detection via the FM4 Measure in Application for Helicopter HUMS (Health Usage and Management System). Proceedings of the Aerospace Conference 2003; 7: 3313 - 3326.

8. Zakrajsek JJ. An Investigation of Gear Mesh Failure Prediction Techniques. Ames Research Center 1989; NASA/TM 89-C-005.

9. Hochmann D, Baringer G. Analytical Mechanical Diagnostic Benefits: Case Studies. IEEE Aerospace Conference 2006.

10. Lebold M, McClintic K, Campbell R, Byington C, Maynard K. Review of Vibration Analysis Methods for Gearbox Diagnostics and Prognostics. Proceedings of the $54^{\text {th }}$ Meeting of the Society for Machinery Failure Prevention Technology 2000: 623-634.

11. Guan YH, Lim TC, Shepard WS Jr. Experimental study on active vibration control of a gearbox system. Journal of Sound and Vibration 2005; 282: 713-733.

12. Combet F, Gelman L. Novel adaptation of the demodulation technology for gear damage detection to variable amplitudes of mesh harmonics. Mechanical Systems and Signal Processing 2011; 25: 839-845.

13. McFadden PD. Low frequency vibration generated by gear tooth impacts. NDT International 1985; 18(5): 279-282.

14. Endo H, Randall RB. Enhancement of autoregressive model based gear tooth fault detection technique by the use of minimum entropy deconvolution filter. Mechanical Systems and Signal Processing 2007; 21: 906-919.

15. Blechertas V, Bayoumi A, Goodman N, Shah R, Shin Y-J. CBM Fundamental Research at the University of South Carolina: A Systematic Approach to U.S. Army Rotorcraft CBM and the Resulting Tangible Benefits. The American Helicopter Society Technical Specialists' Meeting on Condition Based Maintenance 2009.

16. Tandon T, Parey A. Condition Monitoring of Rotary Machines. Condition Monitoring and Control for Intelligent Manufacturing, Springer Series in Advanced Manufacturing 2006: 109-136. 
17. Zakrajsek JJ, Townsend DP, Decker HJ. An Analysis of Gear Fault Detection Methods as Applied to Pitting Fatigue Failure Data. Ames Research Center 1993; NASA/TM 92C-035.

18. Crocker MJ. Handbook of Acoustics. Wiley-IEEE 1998.

19. McFadden PD, Smith JD. A signal processing technique for detecting local defects in a gear from the signal average of the vibration. Proceedings of the Institution of Mechanical Engineers 1985; 199(C4): 287-292.

20. Dempsey PJ, Lewicki DG, Lee D. Investigation of Current Methods to Identify Helicopter Gear Health. Ames Research Center 2007; NASA/TM-2007-214664.

21. Rzeszucinski PJ. Data Fusion in Condition Monitoring of Geared Systems. EngD Thesis (submitted). The University of Manchester, UK, 2012.

22. Johnson RA, Bhattacharyya GK, Statistics: Principles and methods. John Wiley and Sons 2009; $6^{\text {th }}$ edition.

23. Rzeszucinski PJ, Sinha JK, Edwards R, Starr A, Allen B. A New Technique for Condition Monitoring of Helicopter Gearboxes. Proceedings of the $10^{\text {th }}$ International Conference on Vibration Problems Supplement 2011: 407-412.

24. Hess AJ, Hardman B, Neubert C. SH-60 Helicopter Integrated Diagnostic System (HIDS) Program - Diagnostic and Prognostic Development Experience. Proceedings of IEEE Aerospace Conference 1999; 2: 473-491. 